\title{
TAGUNG
}

\section{Die europäische Wettbewerbskontrolle nach der Reform: Chancen und Herausforderungen}

\author{
Ingo Schorlemmer und Joß Steinke*
}

Glaubt man einschlägigen Autoren, ${ }^{1}$ fand am 1. Mai 2004 in Europa eine Revolution statt. An diesem Tag traten die Verordnung 1/2003 des Rates ${ }^{2}$ sowie mehrere begleitende Bekanntmachungen in Kraft - ein Paket der Europäischen Union, das die europäische Wettbewerbspolitik modernisieren sollte. An die Stelle der bislang zentralistisch organisierten Wettbewerbskontrolle durch die Europäische Kommission trat ein stärker dezentral ausgerichtetes System, bei dem die nationalen Kartellbehörden eine größere Rolle spielen. Sie dürfen fortan europäisches Recht direkt anwenden, inklusive der Möglichkeit, Unternehmen oder ganzen Unternehmensgruppen Ausnahmen von den kartellrechtlichen Bestimmungen nach Art. 81 Abs. 3 EG-Vertrag zu gewähren. Bislang hatte dieses Recht allein die Kommission. Außerdem erlosch das Anmeldesystem für Absprachen zwischen Unternehmen sowie für Fusionen, das zu einer Überlastung der Kommission geführt hatte. Um die Kohärenz der Entscheidungen unter EG-Recht sicherzustellen, schufen Kommission und Mitgliedstaaten das „European Competition Network" (ECN), das sich aus der Generaldirektion Wettbewerb sowie den 27 nationalen Kartellbehörden der Mitgliedstaaten zusammensetzt.

\section{The European Anti-Trust Rules - The 2003 Reform and Its Consequences}

Internationaler Workshop des Instituts für

Politische Wissenschaft der FriedrichAlexander-Universität Erlangen-Nürnberg und des Arbeitskreises Europäische Integration (AEI)

Mit Unterstützung des Bayerischen Hochschulzentrums für Mittel-, Ost- und Südosteuropa (BAYHOST), der Dr. German

Schweiger Stiftung, der Deutschen

Forschungsgemeinschaft (DFG) sowie der Europäischen Kommission

Nürnberg, 7./8. März 2008

Wissenschaftliche Leitung:

Prof. Dr. Roland STURM, Universität ErlangenNürnberg

Introduction: The 2003 reform of European anti-trust policies - a social science perspective Prof. Dr. Roland STURM, Universität ErlangenNürnberg

Recent discussions in competition policy after Council Regulation 1/2003

The more economic approach in European competition policy

Prof. Dr. Justus HAUCAP, Universität ErlangenNürnberg; Mitglied der Monopolkommission

* Dipl.-Pol. Ingo Schorlemmer, Wissenschaftlicher Mitarbeiter im DFG-finanzierten Forschungsprojekt „Netzwerke in der europäischen Kartellkontrolle“, Institut für Politische Wissenschaft, Friedrich-Alexander-Universität Erlangen-Nürnberg.

Dipl.-Pol. Joß Steinke, Wissenschaftlicher Mitarbeiter im DFG-finanzierten Forschungsprojekt „Netzwerke in der europäischen Kartellkontrolle“, Institut für Politische Wissenschaft, Friedrich-Alexander-Universität Erlangen-Nürnberg.

1 Lee McGowan: Europeanization unleashed and rebounding: assessing the modernization of EU cartel policy, in: Journal of European Public Policy 6/2005, S. 986-1004; Kris Dekeyser/Maria Jaspers: A New Era of ECN Cooperation, in: World Competition 1/2007, S. 3-23.

2 Verordnung (EG) Nr. 1/2003 des Rates vom 16. Dezember 2002 zur Durchführung der in den Artikeln 81 und 82 des Vertrages niedergelegten Wettbewerbsregeln, in: Amtsblatt der Europäischen Gemeinschaften, Nr. L 001 vom 4. Januar 2003, S. 1-25. 
Knapp vier Jahre nach der Reform fragte ein internationaler Workshop an der Universität Erlangen-Nürnberg nach den Konsequenzen dieser ,Revolution“. Wie funktioniert das Netzwerk der europäischen Kartellbehörden angesichts divergierender Verständnisse von Wettbewerb? Welche Erfahrungen haben nationale Kartellbehörden aus den alten und den neuen Mitgliedstaaten mit der Reform gemacht? Und was bedeuten die aktuellen Diskussionen um den ,more economic approach ${ }^{*}$ und eine private Kartellrechtsdurchsetzung für die europäische Wettbewerbspolitik?

Roland Sturm entwarf zu Beginn des Workshops mögliche Zukunftsszenarien des ECN. Ausgehend vom Status quo stellte er ein Szenario, in dem sich die Zusammenarbeit weiter verbesserte, einem Szenario mit einer Verschlechterung gegenüber. Bislang zeichne sich das Netzwerk durch einen Diskurs über Fälle, durch informelle Kontrolle und Verhandlungen und auf Kooperation basierende Steuerungsformen aus. Solle die Reform tatsächlich so funktionieren, wie das im Modernisierungspaket angelegt sei, müsse nun das Ziel sein, eine gemeinsame Wettbewerbskultur zu erreichen. Eine solche würde nicht nur zu einer Kohärenz der Entscheidungen aller am Netzwerk beteiligter Kartellbehörden führen, sondern auch die Kommission entlasten, da sie die einzelnen Entscheidungen nicht mehr überwachen müsse. Bestenfalls könne das Netzwerk sich selbst steuern. Rückschritte zum bisher Erreichten würden hingegen dazu führen, dass das Netzwerk lediglich formalisierte Routine anwende und zu einem rein administrativen Netzwerk degeneriere. Die Kommission wäre dann gezwungen, das Netzwerk zentral und machtbasiert zu kontrollieren.

Der ,more economic approach ‘ - neues Leitbild der europäischen Wettbewerbspolitik?

Die Diskussion um den ,more economic approach' in der Wettbewerbspolitik griff Justus Haucap auf. Dieser Ansatz solle vor allem die Qualität der Kartellentscheidungen verbessern, indem mehr Informationen in Be-

\begin{abstract}
"More economics" - but "which economics"? On the selection of the "relevant" theories and paradigms

PD Dr. Oliver BUDZINSKI, Universität Marburg

The role of private litigation in the enforcement of European competition law under Regulation $1 / 2003$
\end{abstract}

Prof. Dr. Andreas FUCHS, Universität Osnabrück

The European Competition Network (ECN) from scientific and practical perspective

The network concept in social sciences and the ECN

Prof. Stephen WILKS, Universität Exeter; Mitglied der britischen Competition Commission

Analyzing the ECN - preliminary results of a research project

Joß STEINKE, Universität Erlangen-Nürnberg

What is behind the ECN? A general evaluation Dorothe DALHEIMER, Generaldirektion Wettbewerb, Europäische Kommission, Brüssel

ECN in practice: New commitments, new projects, new solidarities - The experience of the Conseil de la Concurrence

Anne KRENZER, Conseil de la Concurrence, Paris

The national anti-trust policy after Council Regulation (EC) No 1/2003

Experiences in Germany

Dr. Konrad OST, Bundeskartellamt, Bonn

Impact on modernisation in the UK-Views from an OFT official

Steven PREECE, Office of Fair Trading, London

Polish experiences after the 2003/2004 reform

Wojciech DORABIALSKI, Urząd Ochrony Konkurencji i Konsumentów, Warschau

Experiences in Hungary

József SÁRAI, Gazdasági Versenyhivatal, Budapest

Experiences in the Czech Republic

Dr. Michal PETR, Úřad pro ochranu hospodářské soutěže, Brno

tracht gezogen werden als bislang. Neue Erkenntnisse hätten zu einer Neubewertung von bestimmten Handlungsweisen geführt. Es habe sich gezeigt, dass das Verbieten solcher 
Praktiken, die früher negativ gesehen wurden, unter bestimmten Voraussetzungen schädlich sein kann. Der ,more economic approach biete neue Methoden, definiere aber auch neue Ziele der Wettbewerbspolitik. Habe der EG-Vertrag den freien und ungestörten Wettbewerb propagiert, betone die Kommission heute Konsumentenwohlfahrt als Ziel. Wettbewerb wäre damit Mittel zum Zweck, eine Entwicklung, die gerade in Deutschland stark diskutiert werde. Haucap zufolge ist die Kernfrage, was in jenen Fällen geschehe, in denen Wettbewerb die Wohlfahrt nicht erhöht oder gerade eine Einschränkung des Wettbewerbs sie erhöht. Haucap fragte auch, bis zu welchem Grad das Einschränken des Wettbewerbs überhaupt noch erlaubt sei. Er sprach sich für differenziertere Regeln aus. Dabei müsse diskutiert werden, wie ausgefeilt die Regeln sein und wie häufig sie sich ändern sollten. Gerade auch weil der ,more economic approach ' eine Effizienzverteidigung zulasse, sei eine Differenzierung der Regeln unerlässlich. Haucap plädierte außerdem für eine Einzelfallprüfung anstelle starrer Per-se-Regeln.

Für mehr Ökonomie in der Wettbewerbskontrolle sprach sich auch Oliver Budzinski aus. Es stelle sich jedoch die Frage, was das Ziel von Wettbewerbspolitik sein solle. Kartellrechtliche Entscheidungen in Europa seien schon immer ökonomisch basiert gewesen, nur habe es immer jeweils eine Wettbewerbstheorie gegeben, auf deren Annahmen diese Entscheidungen basierten. Der ,more economic approach ' als moderne Oligopoltheorie könne jedoch als Metatheorie verstanden werden, die zu unterschiedlichen Graden mehrere Wettbewerbstheorien in sich vereine. Harvard School und Chicago School hätten sich stark niedergeschlagen, Ordoliberalismus und die österreichische Schule kaum. Kein Paradigma sei den anderen überlegen. Insofern könne keine Auswahl getroffen werden und im ,more economic approach "kämen daher unterschiedliche Wettbewerbsparadigmen zum Einsatz.

Eine zweite Diskussion in der europäischen Wettbewerbspolitik fragt, inwieweit die be- hördliche Kartellrechtsdurchsetzung mit einer privaten ergänzt werden solle. Andreas Fuchs schrieb der privaten Kartellverfolgung eine Doppelfunktion zu: Zum einen diene sie dem Schutz individueller Rechte, zum anderen sorge sie für einen ungestörten Wettbewerb. Sie sei besonders wichtig, da sich die Behörden aufgrund begrenzter Ressourcen vor allem auf wichtige und Hardcore-Kartelle konzentrierten. Zudem sei mit der Abschaffung des Anmeldesystems die Ex-ante-Kontrolle weggefallen, was Informationslücken zur Folge habe. Für eine effektive private Kartellrechtsdurchsetzung wäre nach Ansicht von Fuchs eine indirekte minimale Harmonisierung der nationalen Rechtssysteme in Bezug auf private Rechtsmittel notwendig. Fuchs sah allerdings auch einige Schwierigkeiten. Offen seien beispielsweise noch die Fragen, wie Beweise erbracht werden sollen oder wie private und öffentliche Kartellrechtsdurchsetzung koordiniert werden könnten, um Doppelbestrafungen auszuschließen.

Von der Wissenschaft zur Praxis - die Evaluation des European Competition Networks

Der zweite Teil des Workshops stand im Zeichen einer näheren Untersuchung des ECN. Stephen Wilks stellte dabei fest, dass innerhalb des Netzwerks deutliche Unterschiede zwischen den Behörden existierten, was an der unterschiedlichen Ausstattung mit Ressourcen abgelesen werden könne. Daraus ließe sich zugleich auf einen unterschiedlich starken Einfluss der einzelnen Behörden schließen. Das ECN kümmere sich weniger um Politikgestaltung als um die Rechtsdurchsetzung. Es bestehe ausschließlich aus gouvernementalen Akteuren, kenne also eine exklusive Mitgliedschaft unter einer soft-law-basierten Führung der Generaldirektion. Zwar gebe es nach Ansicht von Wilks einige Problembereiche in der Zusammenarbeit, etwa bei der Einführung strafrechtlicher Konsequenzen für kartellrechtliche Vergehen, insgesamt funktioniere das ECN trotz vielfältiger zentrifugaler Kräfte gut. Diese Stabilität erklärte Wilks mit dem gemeinsamen Diskurs über eine normative 
Basis für eine gemeinsame Wettbewerbskultur und damit, dass die Teilnehmer mithilfe ihrer Partner gegenüber anderen nationalen Akteuren ihre Unabhängigkeit betonen könnten.

$\mathrm{Ob}$ es eine gemeinsame Wettbewerbskultur und einen echten Diskurs darüber gebe, daran zweifelte Joß Steinke, der die vorläufigen Ergebnisse eines Forschungsprojektes über Netzwerke in der europäischen Kartellkontrolle vorstellte. Dass das ECN informale Kontakte und damit den Austausch zwischen den Behörden vereinfacht habe, bestätigte Steinke. Die neue Qualität der Beziehungen sah er als Erklärung für das Ausbleiben von Konflikten im Netzwerk. Das ECN habe ansatzweise hierarchische Strukturen mit einem starken Netzwerkkern, der von Behörden mit reichlich Erfahrung, Ressourcen und mit einem großen Mitgliedstaat im Hintergrund gebildet werde. Zusätzlich gebe es jedoch kleinere und jüngere Behörden, die besonders aktiv und daher auch einflussreich seien. Dennoch sei ein Gefälle bezüglich des Imports und Exports von Wissen innerhalb des ECN festzustellen. Die Generaldirektion sah Steinke als dominierenden Moderator, als Zentrum des Netzwerks. Die nationalen Behörden antizipierten die Position der Generaldirektion in ihren Entscheidungen, außerdem habe sie einen starken inhaltlichen Einfluss auf das Agenda-Setting. Insgesamt erweise sich das ECN als nützliches Kommunikationsinstrument hinsichtlich Fallverteilung und Informationsaustausch. Jedoch gebe es bislang noch keine Hinweise auf systematisches intraorganisationelles Lernen. Das ECN schöpfe sein Potenzial, eine gemeinsame Wettbewerbskultur zu schaffen, noch nicht aus.

Nach der wissenschaftlichen Betrachtung kamen die Praktiker zu Wort. Dorothe Dalheimer stellte den Erfolg des ECN bei der gemeinsamen Verfolgung von europaweiten Kartellen heraus. Sie wies darauf hin, dass die Diskussionen unter den ECN-Behörden zwar durchaus, wie von Sturm und Steinke argumentiert, vor dem Hintergrund einer Hierar- chie stattfänden. Letztlich würden sich aber stets die überzeugendsten Argumente durchsetzen. Dalheimer berichtete, dass die Kommission das ECN als Erfolgsmodell betrachte. Das gelte auch für die Gesamtreform im Zuge der Verordnung 1/2003. Die vor der Umsetzung befürchteten negativen Folgen seien ausgeblieben. So habe es auch keine Klagewelle gegeben. Zwar seien vermehrt Fälle vor das Europäische Gericht erster Instanz getragen worden. Insgesamt sei dieser Anstieg jedoch im Rahmen geblieben.

Auch für Anne Krenzer hat sich das ECN als ein sehr nützliches Instrument der Kooperation zur Angleichung nationaler Vorgehensweisen und Verfahrensregeln erwiesen. Das ECN schaffe neue Verpflichtungen (beispielsweise Informationspflichten), die den Weg in Richtung Kohärenz ebneten, und fördere die Solidarität, beispielsweise indem Untersuchungen im Auftrag von anderen Behörden durchgeführt werden. Auch neue Projekte seien wichtig, erklärte Krenzer. So nutze das ECN die Erfahrungen nationaler Kartellbehörden zur , weichen Harmonisierung ' ihrer Verfahrensregeln in den Arbeitsgruppen des Netzwerks. Ziel sei es, ,best practices' und , benchmarks' zu identifizieren und die eigene Behörde daran zu messen. Diese Möglichkeit werde immer öfter genutzt, der Aufbau einer gemeinsamen einheitlichen Politik komme dadurch gut voran.

\section{Informelle Kontakte spielen die entscheidende Rolle}

Im dritten Teil des Workshops standen die Erfahrungen der nationalen Kartellbehörden mit der Reform im Mittelpunkt. Konrad Ost bezeichnete das neu eingeführte ECN als ein Werkzeug der effektiven Kartellrechtsdurchsetzung und betonte den großen Nutzen des Netzwerks, um informelle Kontakte zu knüpfen und darüber Informationen unkompliziert auszutauschen. Gleichzeitig kritisierte er, dass viele Behörden mit zu wenig Personal ausgestattet seien. Käme auf diese eine steigende Zahl von Anfragen aus dem ECN zu, be- 
stünde die Gefahr einer ,ECN-Müdigkeit". Bezüglich der Diskussionen um neue Ziele und Methoden in der Wettbewerbspolitik verdeutlichte er eine eher skeptische Haltung des Bundeskartellamts. Aus seiner Sicht lassen sich die nationalen Behörden nicht einfach von der Kommission dominieren. Für ihn ist die Generaldirektion nur ein primus inter pares innerhalb des ECN. Ost relativierte auch die Befürchtungen, die es im Vorfeld der Reform in Bezug auf die Kompetenzen der Generaldirektion gegeben hatte. Nach Art. 11 Abs. 6 der Verordnung 1/2003 darf diese ein Verfahren an sich ziehen, woraufhin die nationale Behörde ihre Zuständigkeit verliert. Einen solchen Fall habe es bislang nur ein einziges Mal gegeben.

Auch Steven Preece betonte die gute Kooperation innerhalb des ECN. Positiv sah Preece die ECN-Arbeitsgruppen, die eine gute Möglichkeit zum Austausch und zur Verständigung über die Art und Weise der Zusammenarbeit böten. Zudem hätten die nationalen Behörden über die Arbeitsgruppen größere Chancen, die Richtung der Wettbewerbspolitik zu beeinflussen. Das britische Office of Fair Trading habe kaum Anpassungsprobleme an die neuen europäischen Regeln gehabt. Preece begründete dies mit dem Competition Act von 1998, der das britische Recht dem europäischen bereits angeglichen habe. Schwierigkeiten bereite es, festzustellen, ob ein Fall Auswirkungen auf den zwischenstaatlichen Handel habe - dies ist Voraussetzung für die Anwendung europäischen Rechts. Insgesamt sei die britische Behörde durch die Angleichung an das europäische Recht entlastet worden, weil das Anmeldesystem auch in Großbritannien weggefallen sei. Das Office of Fair Trading sei nun besser in der Lage, eigene Prioritäten zu setzen und sich auf die Fälle mit großer Wirkung zu konzentrieren.

Kartellbehörden der neuen Mitgliedstaaten kämpfen mit hoher Personalfluktuation

Die Erfahrungen der Behörden aus den neuen Mitgliedstaaten standen im Mittelpunkt des letzten Teils des Workshops. Wojciech Dorabialski verwies auf den Personalmangel der polnischen Behörde. Es könnten daher nur wenige Sitzungen auf europäischer Ebene besucht werden. Ein großes Problem sei zudem die hohe Personalfluktuation, was auch die Vertreter der ungarischen und tschechischen, aber auch der britischen Kartellbehörden für ihre Ämter bestätigten. In Polen läge der Grund in den vergleichsweise geringen $\mathrm{Ge}-$ hältern. All dies führe dazu, dass Polen eine passive Rolle im ECN spiele. Nur etwa zehn Prozent der Fälle würden bei der Generaldirektion notifiziert, was vor allem daran liege, dass das polnische Wettbewerbsrecht mit dem europäischen Recht identisch sei und es daher in den Entscheidungen kaum Unterschiede gebe. Zwar profitiere die Behörde vom Informationsaustausch im ECN. Gleichzeitig würden jedoch die umfassenden Informationspflichten in der Anwendung der Art. 81 und 82 EG-Vertrag die Verfahren verlangsamen.

Das ungarische Wettbewerbsrecht, berichtete József Sárai, habe sich schon bei seiner Implementierung 1990 stark am europäischen Recht orientiert. Nach der Aufnahme in die Reihe der Beitrittskandidaten habe Ungarn eine weitere Angleichung implementiert. Das mit dem Beitritt verbundene Modernisierungspaket von 2004 sei eine große Herausforderung gewesen, weil für die neuen Mitgliedstaaten auch neue Aufgaben entstanden seien, wie etwa die Teilnahme am Advisory Committee. Dafür seien die Lernprozesse, die das ECN erleichterte, von besonderer Bedeutung gewesen. Auch nach dem Beitritt zur Europäischen Union habe es Veränderungen des Wettbewerbsrechts gegeben. So habe Ungarn unter anderem bereits die private Kartellrechtsdurchsetzung eingeführt und sich neue Regelungen für Sektoruntersuchungen gegeben. Insgesamt sei das Ziel der im ECN sehr aktiven ungarischen Kartellbehörde eine fortgesetzte Angleichung des materiellen Rechts, der Verfahrensregeln, der Sanktionen und der Kronzeugenpolitik. 
Von einer ganzen Reihe von Problemen bei der Durchsetzung des Kartellrechts in der Tschechischen Republik berichtete Michal Petr. Dazu zählten die begrenzten Erfahrungen der tschechischen Behörde und fragwürdige Gerichtsurteile. All dies geschehe vor dem Hintergrund umfassender gesetzlicher Veränderungen in den Jahren nach dem Beitritt und der Einführung neuer, ,EU-freundlicher' Kronzeugen- und Sanktionsregelungen. Petr berichtete, dass für die tschechische Behörde besonders bei Durchsuchungen von Geschäftsräumen ohne richterliche Verfügung Probleme auftreten könnten und kritisierte die mangelnde Unterstützung der Behörde durch die Polizei. Dennoch habe die Zusammenarbeit im ECN bislang reibungslos funktioniert. Schwieriger gestaltet sich Petr zufolge die Zusammenarbeit mit den Gerichten, vor allem wenn die Behörde nationales und europäisches Recht parallel anwende. Der Rechtsstreit über diese Praxis liege derzeit zur Verhandlung vor dem obersten Gericht.

\section{Fazit}

Vorträge wie auch Diskussion verdeutlichten, dass das ECN informelle Kontakte und Infor- mationsaustauschprozesse zwischen den einzelnen Behörden deutlich erleichtert hat. In den Ämtern haben Lernprozesse eingesetzt, in denen das eigene Vorgehen an europäischen Vergleichsmustern gemessen und bewertet wird. Dieses Lernen könnte jedoch von der hohen Personalfluktuation konterkariert werden. Dennoch: Die Reform hat - so darf nach diesem Workshop angenommen werden - das europäische System der Kartellrechtsdurchsetzung effektiver gemacht. Allerdings verweisen unterschiedliche Prioritätensetzungen und abweichende Definitionen dessen, was mithilfe von Wettbewerb erreicht werden soll, auf die Schwierigkeiten, die noch vor uns liegen. Die Diskussion über den ,more economic approach` könnte zu einer Belastungsprobe werden, weil dieser Ansatz an den Grundfesten des Wettbewerbsverständnisses einiger Behörden rüttelt. Inwieweit die Reform somit tatsächlich zur Generierung und Verstetigung einer gemeinsamen Wettbewerbskultur beiträgt, ist weiter offen. Die enge und unkomplizierte Zusammenarbeit und der rege Austausch der Behörden untereinander könnte jedoch ein erster Schritt hin $\mathrm{zu}$ einer gemeinsamen Basis sein.

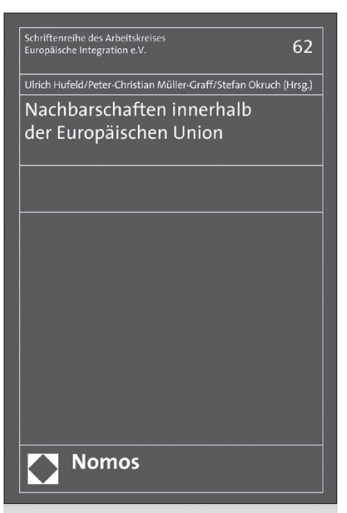

\section{Nachbarschaften innerhalb der Europäischen Union}

Herausgegeben von Ulrich Hufeld, Peter-Christian Müller-Graff und Stefan Okruch 2008, 273 S., brosch., 59,- €, ISBN 978-3-8329-2994-7 (Schriftenreihe des Arbeitskreises Europäische Integration e.V., Bd. 62)

Die Nachbarschaften an den Binnengrenzen der Europäischen Union sind ein interdisziplinäres Thema dauerhafter integrationswissenschaftlicher Aktualität. Der Band ist Ergebnis einer multidisziplinären Konferenz zum Thema. 